\title{
Model similarity evidence and interoperability affinity in cloud-ready Industry 4.0 technologies
}

\author{
G. Pedone ${ }^{\mathrm{a}, *}$, I. Mezgár ${ }^{\mathrm{a}}$ \\ ${ }^{a}$ Research Laboratory on Engineering $\&$ Management Intelligence, Hungarian Academy \\ of Sciences, Kende st. 13-17, Budapest, Hungary, 1111
}

\begin{abstract}
Cloud computing is revolutionizing IT environments in most fields of economy. Its service-based approach enables collaboration and data exchange on higher level, with better efficiency and parallel decreasing costs. Also manufacturing environments can benefit from cloud technology and better fulfill fast changes in market demands, by applying diverse cloud deployment models and by virtualizing manufacturing processes and assets into services. As cloud becomes the basis of most innovative manufacturing IT systems, its future role in Cyber-physical Production Systems has to be properly investigated, as their interoperability will play a role of vital importance. In this paper, after a brief introduction to cloud criticality and cloud-based manufacturing, the mutual conceptual similarities in modelling distributed industrial services of two of the major standardization frameworks for industrial Internet architectures are presented: the Industrial Internet Reference Architecture (IIRA) and the Reference Architectural Model Industrie (RAMI 4.0). It is also introduced how their integration feasibility finds a strong affinity in specifications of the Open Connectivity Unified Architecture, a service-oriented architecture candidate to the standardization of Industrial Internet of Things based manufacturing platforms. Finally, the preliminary architecture of a prototype Smart Factory is presented as a case study.
\end{abstract}

Keywords: Cloud Computing, Cloud Manufacturing, Industry 4.0 Interoperability, IIRA, RAMI 4.0, OPC UA

\footnotetext{
*Corresponding author

Email addresses: gianfranco.pedone@sztaki.mta.hu (G. Pedone), mezgar.istvan@sztaki.mta.hu (I. Mezgár)
} 


\section{Introduction}

Based on the evolution of the information and communications technologies, a new "digital" economy has reached a point where it can be called the forth revolution, and where Cyber Physical Systems (CPSs) are interlinked through real and virtual objects and processes. This phase is featured as a deep interdisciplinary integration of technologies in digital, physical and biological world (Schwab, 2015). Manufacturing industry also belongs to those sectors which are basically changing in all of their component systems as new paradigms are consolidating (e.g. additive manufacturing, nanotechnology, cloud based manufacturing) and the integration of subsystems is becoming crucial for interoperability. The European Commission has launched an initiative named "Digitising European Industry" which focuses on speeding up the standardization on five priority areas: $5 \mathrm{G}$, cloud computing, internet of things - IoT, data technologies and cyber-security (EC, 2017). In USA the National Science Foundation released a call on "Cyber physical Systems" in December 2016 that covers, among others, the research areas of IoT, CPS Security and Privacy, Real-time Control and Adaptation and Manufacturing (NSF, 2016). The high-tech ICT-based strategic program of the German government, called "Industrie 4.0", primarily focuses on manufacturing and describes the up-to-date automation and data exchange in manufacturing technologies, including Cyber-Physical Systems (CPS), Internet of Things (IoT) and Cloud Computing (CC). According to a statistics from Weins (2017), the $95 \%$ of US firms use CC technology, while the applications of Cloud-based Manufacturing (CM) are trying to make use of CC technology by mirroring its service orientation and deployment approach. The Industrial Internet Reference Architecture (IIRA) and the Reference Architectural Model Industrie (RAMI 4.0) are two of the major standardization frameworks for industrial Internet architectures, which aim at extending industry interoperability through a high level of abstraction and common use case characteristics (in the case of RAMI 4.0 also by providing features and patterns derived from the manufacturing domain). The aim of this paper is to show how both technologies share conceptual similarities in modelling distributed industrial services, and also how their integration feasibility finds relevant affinity in the specification of OPC Unified Architecture (OPC UA), as one of the possible candidate architectures for IIoT-based service standardization (Hein, 2017). The paper finally presents a case study containing the preliminary architectural of a prototype smart factory. 


\section{Motivations and related work}

Based on opinions of industrial experts in cloud environments, the greatest challenge facing longer-term adoption of $\mathrm{CC}$ might not be security, but rather interoperability and data portability (Weissberger, 2011), due to the exponential growth of Internet-connected devices involved in new business models (Fabode, 2016). Industrial Internet Consortium and "Plattform Industrie 4.0" in March 2016 announced common efforts to align RAMI 4.0 and IIRA but they did not agree on an ultimate solution. Today CM interoperability is in the focus of current research topics and this paper aims at showing that Industry 4.0 (I4.0) service standardization is not a mere problem of communication and protocols but taking into consideration interoperability already starts at design level: this permits to realize a significantly higher flexibility and adaptability of production systems. I4.0 is all about information and data exchange: between people, machines, materials and systems. But while standardization is indispensable in communication, machines and systems should be helped in interpreting the meaning of the communication and that is where interoperability comes in: interoperability is about the meaning of the contents of exchanged data.

Several papers can be found in literature in this field but their argumentations are not fully in scope with the ones presented in this paper. In Gøtze (2016), for example, a major attention is given to RAMI 4.0, recalling that possible generalization of reference architectures is not an I4.0 specific topic as it has always seen important attempts in industry starting from the early 1990s. In Uslnder and Epple (2015) authors essentially focus on RAMI 4.0 and its nature of service oriented architecture (SOA). In Pai (2017) author provides an interesting comparison of both architectures but this description is customized to specific application of the industrial assets.

On the other hand, this paper's specific contributions aim at:

- presenting general indications of functional mapping between IIRA and RAMI 4.0;

- highlighting a modelling link for interoperability between the concepts of digital twin in IIRA and the Administration Shell in RAMI 4.0 (RAMI 4.0 AS);

- providing evidence of the affinity between RAMI4.0 AS and OPC UA (as a possible candidate to bridge I4.0 service infrastructure) in modelling the underlying physical environment; 
- establishing a development pattern for interconnected physical components and services in I4.0 manufacturing architectures.

\section{Cloud, Cloud Manufacturing and Factories of the Future}

The future of manufacturing will probably depend on the conceptualization of new architectures, which cannot be handled in a traditional manner as smart devices, or in general smart-X-objects (including humans), smart connected assets intend to communicate directly with each other. The development of new interaction models will be the crucial aspect of future production strategies.

\subsection{Cloud characteristics and deployment models}

$\mathrm{CC}$ is in general an IT architectural model where computing services (both hardware and software) are abstracted and delivered to customers over the Internet, on-demand, in a self-service fashion, independent of device and location (Marston et al., 2011). Cloud model embodies unique characteristics and can have various service and deployment models: while service models are typically an end-user's perspective in CC industry, different delivery models refer to different layers of the $\mathrm{CC}$ architecture. The most common form of CC is Software as a Service (SaaS), in which the application runs on the vendors infrastructure and is recognized as a service. The consumer is unaware of the application providers infrastructure and complexity. A Platform as a Service (PaaS) facilitates the development of applications without the cost and complexity of buying and managing the underlying hardware and software layers, like operating system, network, and servers, and development tools. Infrastructure as a Service (IaaS) offers also storage, network and computational capabilities as a service.

Container as a Service ( $\mathbf{C a a S}$ ) is a form of cloud-based virtualization in which so-called container engines, orchestration and the underlying computing resources (application, operating system dependencies, libraries, and so forth) are delivered to users as a service from a cloud provider. Serverless computing (or Functio as a Service - FaaS) is the highest technology level today, where the whole application, with all of its business logic, is implemented as functions and events at cloud level. Manufacturing specific Hardware as a Service (HaaS) is a technique for consistently describing and serving equipment and its functionality, behaviour, structure, etc., and represents one of the major challenges in service implementation description of core physical 




Figure 1: Cloud deployment models with property-specific service instantiation

equipment in the context of Manufacturing as a Service (MaaS). Cloud in manufacturing is expected to provide a solid support for service-oriented environments, simulations and global services. For a comprehensive list of key players in the CC industry can be found in Marston et al. (2011).

Essential properties of cloud-based systems can be classified according to $\mathrm{Xu}$ (2012), Zissis and Lekkas (2012), and Wang and Xu (2013) into core, business, enterprise and manufacturing clouds. Resource abstraction, selfservice-centricity, rapid elasticity, multi-tenacity, load-balancing and virtualization are basic characteristics of CC. Business-relevant properties comprise quality-of-service, service level agreement, user experience, fault-tolerance, auditability and certifiability. At an enterprise level, interoperability, deployment models, security and business process management increase the complexity of $\mathrm{CC}$ requirements.

Cloud hosting deployment models are mainly distinguished by the proprietorship, size and access and are classified in public cloud (the cloud owner provides Internet-based public services on predefined rules, policies, and a pricing model); private cloud (benefits of a public cloud but decreased security concerns as used "in-house" by the company); community cloud (organizations having similar requirements and concerns share the $\mathrm{CC}$ and a third-party service provider is responsible for providing the required infrastructure of the CC); and hybrid cloud (a combination of two or more different public, private, or community clouds). In hybrid clouds business- 
and mission-critical services and sensitive data are kept unpublished, while non-critical services are published for others to share and use. Illustration on Figure 1 depicts common cloud deployment models with service-critical instantiation orientations.

Besides obvious benefits of CC there are still significant barriers for its adoption; e.g. according to Zissis and Lekkas (2012) and Lee (2008): missioncritical services in enterprises need to be locally reinforced, in order to ensure continuity in the execution of business processes. Consulting companies reported how current cloud services may not be cost-effective for larger enterprises which have achieved best efficiencies from their own computing infrastructure (Bommadevara et al., 2016).

\subsection{Cloud standardization}

Cloud service providers usually have own approach on interactions and cloud-APIs required to users but complex applications on the cloud require adequate standards, in order for organizations to consolidate their IT systems in the cloud and realize productivity increase. To-day more than 15 different groups, committees and organizations have established a Wiki site for Cloud Standards Coordination (Cloud-standards.org, 2018), whose goal is to document activities from various Standards Development Organizations (SDOs) and leading technology. In general, there are different major approaches to solve interoperability challenges in networked enterprises: integrated - a common format for all models; unified - common format at meta-level and mapping between models; and federated. Our scope addresses the federated one, in which no common format exists and partners have to share ontology to map concepts at semantic level. The detailed description of the above methods can be found e.g. in Chen et al. (2008). Trend and issues for enterprise integration and interoperability in manufacturing systems are presented in detail in Panetto and Molina (2008).

\subsection{Cloud Manufacturing}

The idea of Cloud manufacturing has been developed upon the cloud computing technology (Li et al., 2010): the basic idea is to apply cloud computing architecture, tasks and service structure to manufacturing systems. The name of the paradigm varied from Manufacturing Cloud - MCloud (Zhang et al., 2012) to Cloud Based Manufacturing - CBM (Wu et al., 2014) but the content behind the words is the same. There are at least three areas where 
$\mathrm{CC}$ can be applied in manufacturing companies, in the form of SaaS/FaaS solution:

- Data collection and analytics: primarily focused on equipment operations and management, Big Data in manufacturing strengthen the utilization of a common data model to combine structured business data (like inventory transactions and financial transactions) with structured operational data (like alarms, process parameters, and quality events) and unstructured internal and external data (like customer, supplier, Web, and machine data) to discover new insights through advanced analytical tools;

- Inter-factory collaboration with an extended scope: services like supply chain visibility, transportation management, supplier/contract negotiation. Partners can create cloud computing modules to address other manufacturing issues, e.g. supply chain execution, and production scheduling;

- High performance computing and simulation: using digital models to (1) virtually test the products or manufacturing system, (2) understand the business environment better through business intelligence and (3) make decisions. The models are typically highly parallelizable and fit well for a cloud environment.

By using the methods of $\mathrm{CC}$ it is possible to convert production oriented manufacturing processes into service oriented networks by representing single manufacturing assets as offered services . To access manufacturing resources as cloud services, manufacturing platforms generally need to have a layered structure with different levels and content, from manufacturing resources up to enterprise collaboration level. Each of the products life cycle can be represented in different service models (based on IaaS, PaaS, and SaaS). Some examples: the design - in the form of design as a service (DaaS); the manufacturing - manufacturing as a service (MaaS); and simulation - simulation as a service (SIMaaS). Virtualization is the most important phase of transforming a conventional manufacturing system to cloud manufacturing: virtualization means to dynamically divide different resources into virtual units and later combine them flexibly (with other resources) to a logic unit to meet diversified demands as encapsulated services. Some major virtualization approaches described in literature include Zhang et al. (2017) (a service 
encapsulation and virtualization access model) and Ning and Xiaoping (2012) (a cloud manufacturing virtualization framework has been described, which contains three layers: manufacturing resource, virtual description and service encapsulation layer).

\subsubsection{Cloud Manufacturing Interoperability}

Interoperability is an essential requirement for both service providers and enterprises. Interoperable services allow applications to be ported between clouds, or to use multiple cloud infrastructures before business applications are delivered from the cloud. Nevertheless, interoperable solutions make easy migration and integration of applications and data between different cloud service providers $(\mathrm{Xu}, 2012)$. Interoperability can be divided into two main groups in cloud-based systems/applications: i) the interoperability of the cloud systems itself (cloud layer) and ii) the interoperability of the applications (application layer, industry- and HW/SW specific). Yadgarova and Taratukhin (2016) introduced an integrated framework for building cloudbased manufacturing environment which allows developing production systems as adaptive distributed systems with virtual cloud, simulation and equipment interaction dependency model. Mourad et al. (2016) identified interoperability as "a key enabler for cloud manufacturing". They proposed a framework called $C$-MARS for the realization of interoperability across heterogeneous computer aided manufacturing systems (handling CAD and NC files). Using this framework, manufacturing resources can be shared by a large number of clients based on requirements and priorities. A serviceoriented, interoperable cloud manufacturing system (ICMS) is proposed in Wang and $\mathrm{Xu}$ (2013), where service methodologies have been developed to support customer and enterprise users, along with data models describing cloud services and collaborative interactions.

\section{Industry 4.0 Interoperability and Standardization}

The exploitation of I4.0 paradigm requires the availability of adequate information across all engineering and production value chains, which is the result of aggregation and fusion of data from various heterogeneous sources, often under real-time conditions. On the one hand, this comprises a challenge for the provision of an efficient, secure and dependable information management infrastructure. On the other hand, system architects must find solutions to assure interoperability on both syntactical and semantic level 
with reasonable engineering costs. Figure 2 summarizes those aspects which play a key role in the different production systems and shows the extended need for interoperability and security. According to Liu and Xu (2016), these two factors play a fundamental role in I4.0, as the continuous interaction among sensors, mobile devices and humans (through intelligent interfaces) is extremely significant at all levels.

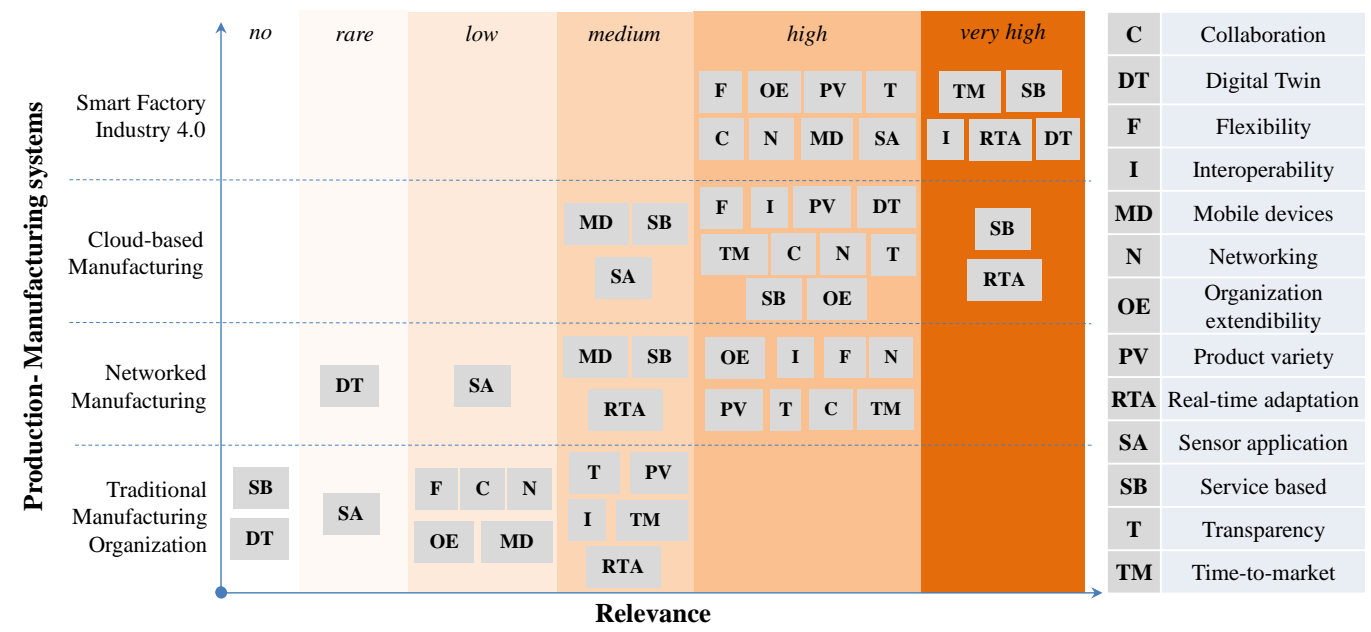

Figure 2: Comparison of the different production and manufacturing systems

Global networking of production resources and processes, as well as the use of globally interconnected applications crucially require the adoption of unified standards. Two major proposals for this standardization are introduced in the following, which are "de-facto" considered as reference guidelines in conceiving future business organizations: the Industrial Internet Reference Architecture (IIRA) and the Reference Architecture Model for Industry 4.0 (RAMI 4.0).

\subsection{IIRA}

IIRA is a standard-oriented open architecture for Industrial Internet Systems (Industrial Internet Consortium, 2015) and aims at extending interoperability in industry and guiding the development of technology standards development. The IIRA is a general purpose architecture with a high level of abstraction to support broad industrial applicability based on use cases defined by the Industrial Internet Consortium (IIC). The architecture framework (IIAF) is based on ISO/IEC/IEEE 42010 (2011) standard specification and codifies conventions and common practices which are at the core 
of IIRA's Internet Information Service (IIS). These architectural concepts comprise concerns (topics of interest in the system), stakeholders (entities having an interest in the system) and viewpoints (conventions framing the description and analysis of specific system concerns). Concerns of an IIS are classified and grouped in four different viewpoints: business, usage, functional and implementation. In this paper the focus is narrowed only on the functional viewpoint because this is more relevant from the point of view of a system interoperability, which is crucial in defining collaboration logics. In functional viewpoint, IIS is decomposed into five sub-domains: control, operations, information, application and business. These domains represent the building blocks of an IIS and illustrate how data and control move across them. The implementation viewpoint describes the general architecture, the technological components of an IIS, and the interfaces, protocols and their behaviours. Implementations are encapsulated under various architectural patterns, all including the three-tier and gateway-mediated edge connectivity pattern (refer to Figure 3 for details).

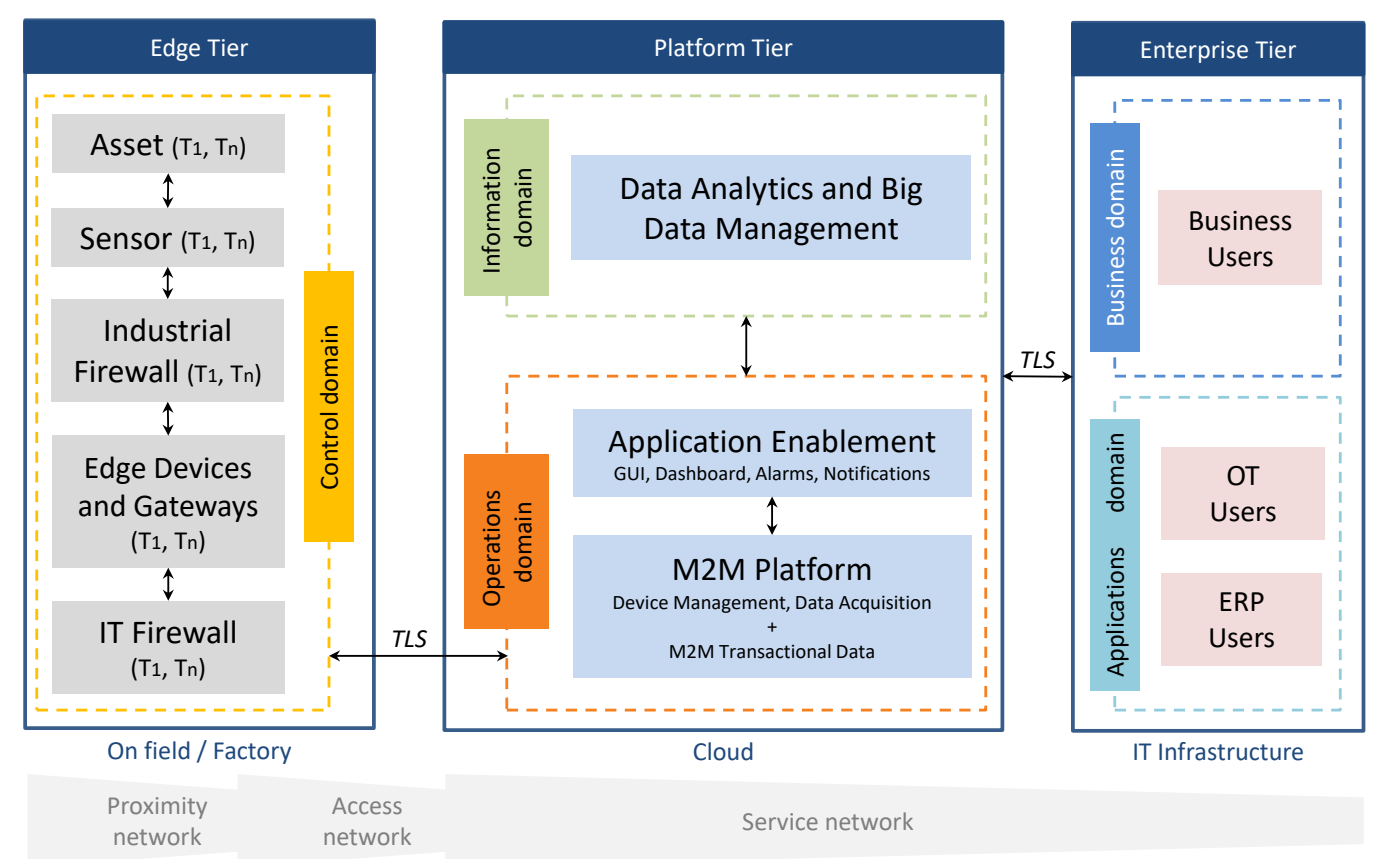

Figure 3: IIRA 3-tier architecture for IIC testbeds (T1,Tn) with functional domains from IIRA and communication networks

IIRA is not a standard but provides guidelines on how a safe, secure and 
resilient IIS can help realize the vision of the IIC. IIRA three-tier architecture can be introduced as follows:

- Edge tier: responsible for collecting data from edge nodes through asset, sensors and gateways, using the proximity network (control domain);

- Platform tier: receives, processes and forwards control commands from the enterprise tier to the edge tier (management functions, data query and analytics for devices and assets);

- Enterprise tier: implements domain-specific applications, decision support systems and provides interfaces to end-users to implement domain specific functionality such as Manufacturing Execution System, Supply Chain Management and Enterprise Resource Planning.

IIRA functional domains can be grouped into:

- Proximity network: connects the sensors, actuators, devices, control systems and assets to a gateway that bridges to other networks, and enables data and control flow between the edge and platform tiers;

- Access network: enables connectivity for data and control flows between the edge and the platform tiers;

- Service network: enables connectivity (usually using Transport Layer Security protocols) between services in the platform and enterprise tiers.

\section{2. $R A M I 4.0$}

Central in RAMI 4.0 is the concept of CPS (analogous to the IIS in IIRA) where autonomy is localized and component systems make decisions on their own. The reference architecture model for RAMI 4.0 (VDI et al., 2015) is the convergence of multiple stakeholder visions on how I4.0 might be realized, based on existing communication standards and functional descriptions. The six layers of the vertical axis of RAMI 4.0 define the nature of IT components in I4.0 (Figure 4): business applications, functional aspects, information handling, communication and integration capability, and ability of the assets to implement I4.0 features. The life-cycle of products, machines, orders and factories are captured along the life cycle and value stream axis (IEC 62890), 
whereas the hierarchy levels represent various functions of enterprise IT and control systems (IEC 62264 and IEC 61512). An important feature of RAMI 4.0 is the identification of objects as instantiated types of a product, asset, software, machine, or even a factory.

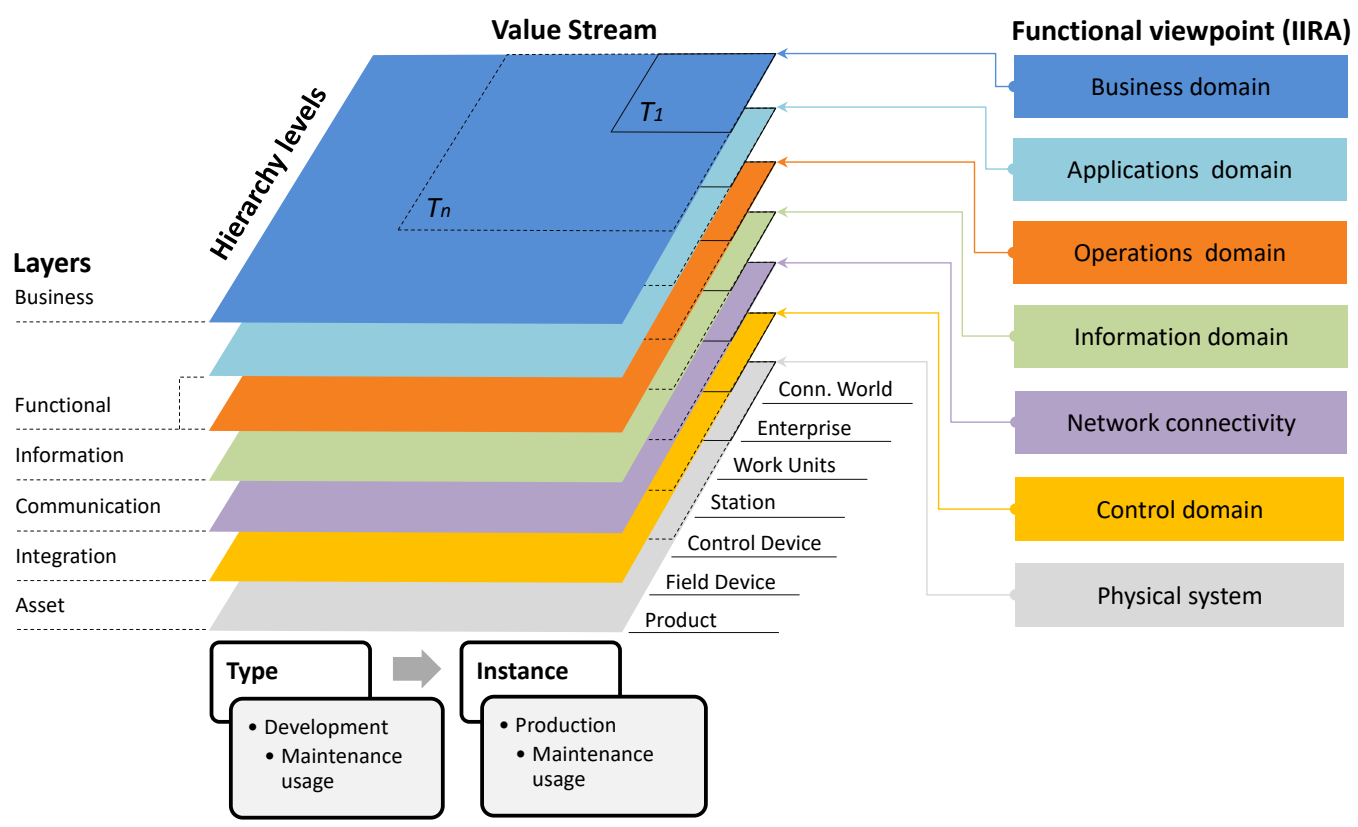

Figure 4: Mapping between IIRA functional viewpoints with IT layers from RAMI 4.0 for testbeds $(\mathrm{T} 1, \mathrm{Tn})$

\subsection{Industrial interoperability: IIRA versus RAMI 4.0}

Interoperability can be defined as the ability of two or more systems to mutually exchange and understand information. It is realized by the implementation of standards and can be ascertained at various levels in enterprises: technical, syntactic, semantic, conceptual or functional and business. The three-tier architectural pattern of IIRA shows distinctive features with RAMI 4.0 architecture when addressing interoperability at their functional level. This indicates that applications might benefit from the cross-implementation of both of these industrial Internet architectures, which provide a fully specified but implementation independent model. More details are presented in the followings.

Fundamental to IIC is the concept of Digital Twin, the computerized (often cloud-based) counterpart of physical assets, which uses data from sensors, 
actuators, power supply and network interfaces installed on physical objects to represent their near real-time status, working condition (properties and states) or position (a typical example is the use of 3D modelling to create a digital companion of the physical objects to be projected into the digital world). Efficient schedule of operations and predictive maintenance of assets are also possible using digital twins. An important aspect of the digital twin is that it can be adapted for different environments to take advantage of data generated by other virtual machines in the ecosystem, according to different designs and requirements.

The central concept in RAMI 4.0, on the contrary, is built around the I4.0 compliancy of components, such as product, asset, software, or machine that be, referring to as objects that have the ability to communicate independently, by using I4.0 compliant communication (Figure 5). Non-I4.0 compliant components are made I4.0 compliant by deploying an Administration Shell (AS), which essentially provides a virtual representation and a description of the entire life-cycle of the object or asset. It corresponds to the IIC digital twin of an asset in the I4.0 domain, and contains its lifecycle, technical functionality, and even procedures for sensor data integration and monitoring. Each product, in order to be integrated into an Industry 4.0 network, needs an Administration Shell, which serves as the networks standardized communication interface.

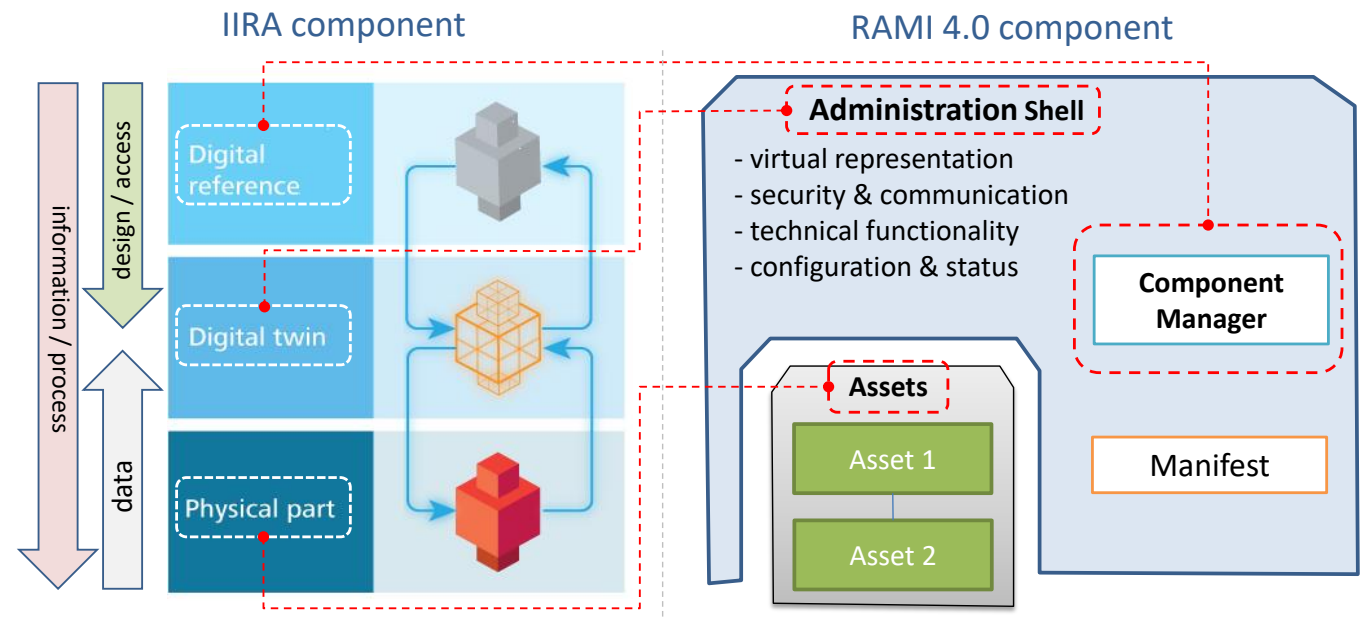

Figure 5: Conceptual mapping between IIC "digital twin" and RAMI 4.0 "Administration Shell"

Two elements play a key role in the definition of an AS: the Virtual 
Representation (VR) and Technical Functionality (TF). The Manifest is an important part of the VR and can be seen as a directory of the VR data and a container of the I4.0 component meta-information, as well as the link to other assets and security indications. The Component manager (CM) is the link of the I4.0 component to ICT technical services and allows external access to VR and TF. The CM can thus connect in a Service-oriented Architecture (SOA) or deploy the AS into a repository. Based on this functional view (illustrated also on Figure 5) the "physical part" of IIRA components can be similarly handled as assets in the AS, the "digital reference" shows analogous functionality to CM, and the "digital twin" in IIRA evidences a role similar to the AS in RAMI 4.0. This does not mean that the two architectures are equivalent but it is clear that there is a sort of functional "alignment" in the abstraction, modelling and provision of physical asset services to the external world. It is worth to underline how the primary objective of the AS remains the standardized integration of assets/things within I4.0 by means of interleaved I4.0 components, whereas the "digital twin" generally aims at benefiting from the virtualization of physical assets in simulations and digital augmentation of the environment.

In preliminary results from IIC \& Plattform Industrie 4.0 (2017), the authors showed how Industrial Digital Thread and Asset Efficiency, two specific paradigms for realizing testbeds in connected industrial organizations (Infosys \& IIC, 2015a,b), can be applied to evidence a mapping between IT layers in RAMI 4.0 and functional domains in IIRA. In Figure 4 an illustration of IIRA 3-tier architecture is reported in the case of arbitrary testbeds (T1, Tn). ICT levels configuration in the factory field, along with the functional viewpoints, the associated functional domains from IIRA, and all relevant communication networks associated with each tier are also depicted. Moreover, a mapping between IIRA functional viewpoints with IT layers associated to RAMI 4.0 architecture for arbitrary testbeds are also reported on previous Figure 4. IIoT solutions, such as testbeds relying on a SOA, can be considered to be semantically interoperable between the two architectures at a functional level.

\subsection{Link between RAMI 4.0 and OPC Unified Architecture}

Both IIRA and RAMI 4.0 do not indicate any specific solution for developing the infrastructural layer underlying system communications and both indicate OPC UA as a strategic player in the realization of industrial services interoperability. But while IIRA refers to it as conceivable solution, 
in this section it is introduced how RAMI 4.0 explicitly shows a conceptual correspondence with OPC UA in modelling information and services, too.

OPC UA is a product of the OPC Foundation (2015), an industry consortium which creates and maintains standards for open connectivity of industrial automation devices and systems. OPC UA is a service-oriented, platform-independent standard whose specification is organized into several documents related to concepts, security model, address space model, services, information model, mappings, profiles, data access, alarms and conditions, programs, historical access, discovery and aggregates. OPC UA defines the sets of services that servers can provide, mapped onto different communication protocols and in which data can be encoded in XML/text and UA Binary. Transport protocols support TCP, SOAP/HTTP and HTTPs. OPC UA enables interoperability by mapping concepts between property sets from different domains and/or frameworks: the more aligned is the conceptual modelling of functions (or services) the more natural will be the mapping between referenced concepts and their direct use. Similar condition has been discovered between OPC UA information model and RAMI 4.0 Administration Shell (RAMI 4.0 AS), in consideration of their service modelling analogies (illustration in Figure 6 serves to explain the following concepts):

- RAMI 4.0 AS structure: the data in the AS (Header) is specified in terms of requirement properties; the component manager (Body) administers sub-models which have hierarchically organised properties, referring to individual data and functions of the physical assets through views over the properties. Properties of all sub-models constitute the Manifest of the AS, which can hence serve as a discoverable table of contents of all data and functions. Finally, I4.0-compliant service-oriented API make methods (intended as a composition of functions in submodels) of the CM available externally;

- OPC UA information model: servers contains the definition of real objects (physical or software) accessed from the Address Space. Objects, in turn, are modelled in terms of Views (business, operational or permission based aggregation of elements), Attributes, Variables, Methods and relationships to other Objects. Elements of models are represented in the Address Space as Nodes, assigned to classes representing different aspects of the objects behaviour. Methods are functions similar to methods of a class in Object Oriented Programming, whose scope and behaviour is specified in terms of attributes and/or variables data, 
or eventually of other methods (in other nodes). Methods are orchestrated in order to define service models, invoked by using the proper discovery and service calls.
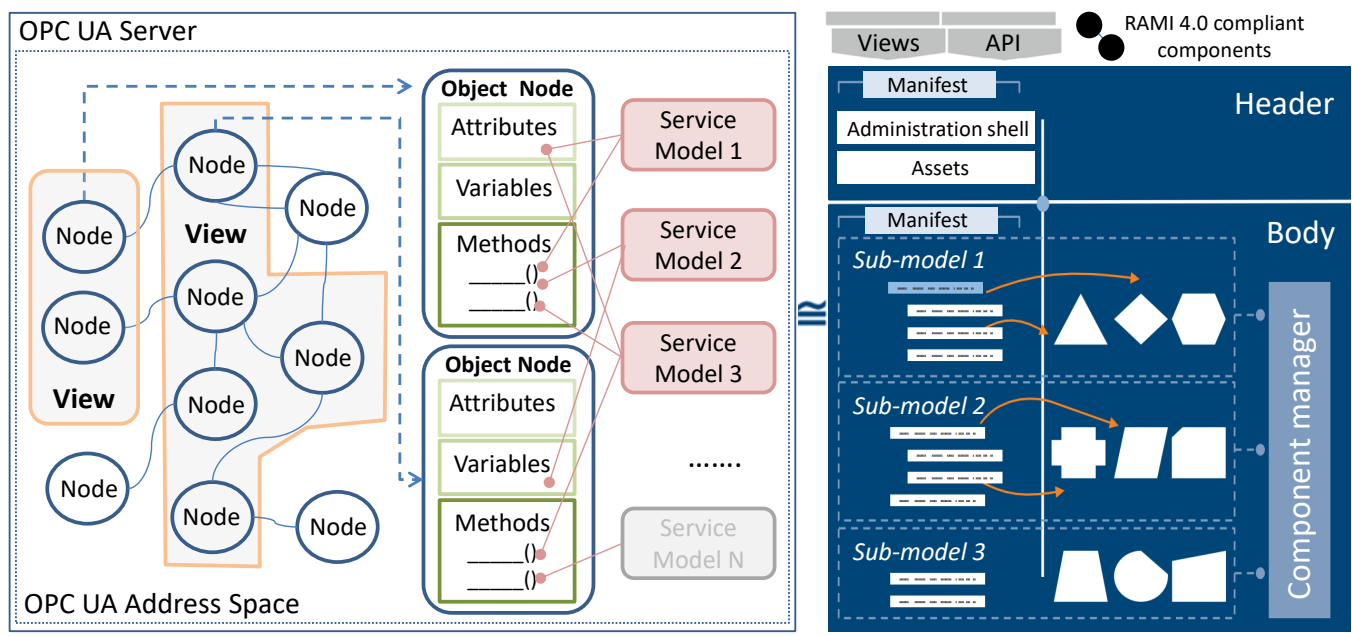

Figure 6: Service modelling analogies between OPC UA server and RAMI 4.0 Administration Shell

It is worth to note, at this point, that both methodologies not only assign a similar role and meaning in the specification of fundamental concepts (for instance assets vs. nodes; assets properties vs. object attributes and variables; sub-models vs. object methods composition and orchestration; component manager vs. address space) but for specific functionality they share even the same terminology, like in the case of views. An example of effective ongoing initiative demonstrating the integration compatibility of OPC UA into RAMI 4.0 AS is the Open Asset Administration Shell, an Open Source Project (openAAS) which aims at implementing an OPC-UA-based AS (it was expected by the end of 2017).

\subsection{RAMI 4.0 and IIRA layers in the Cloud: a possible set of connected functionality}

Figure 7 depicts a possible realization for the integration of the multiple layers in the case of a hypothetical manufacturing ecosystem. In this case different factories are modelled with different methodologies (in the example one factory with RAMI 4.0 and one factory with IIRA), conveying their 
infrastructural virtualization into a connected, cloud-based uniform service orchestration interface.

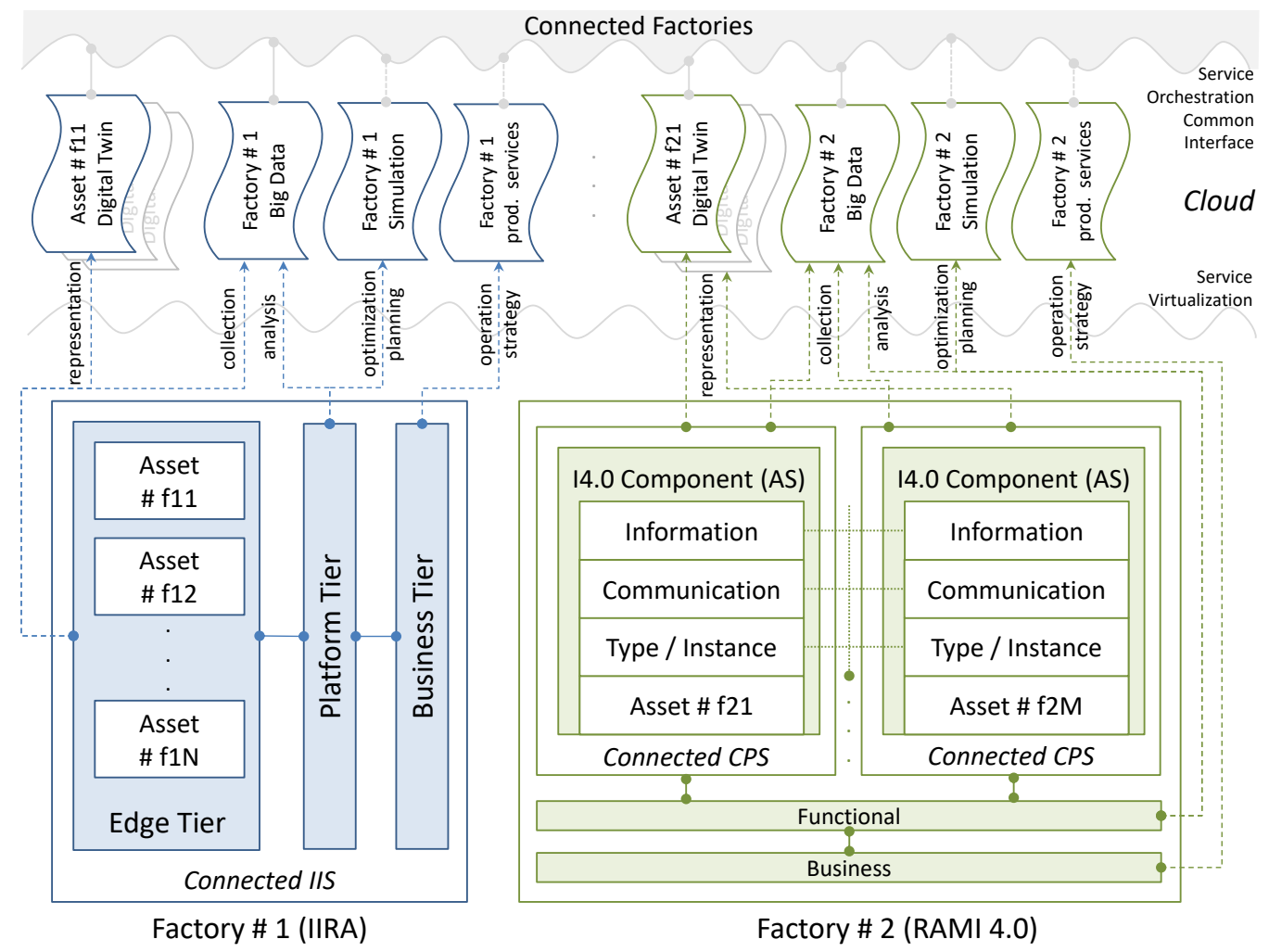

Figure 7: Example of virtualized and connected services from RAMI 4.0 and IIRA layers in the case of a cloud-deployed manufacturing environment

Figure 7 shows how, in a (methodologically) heterogeneous distributed manufacturing ecosystem, every asset (shop-floor components, computational resources, monitoring and control systems, human operators, etc.) participating in the IoT connected network (f11 to f1N for Factory 1; f21 to f2M for Factory 2) can be implicitly connected to its asset lifecycle, which is in turn exposed in terms of cloud-able services (like big data, simulation, resource planning, dashboard, etc.) through the virtualization of layer-specific functionality coming from each architecture: asset representation, data collection and analysis, optimization of processes, planning, operation and strategy. Paradigms such as digital twin (originally associated with factory assets virtualization in IIRA and which finds its counterpart in RAMI 4.0 AS) and 
service virtualization (associated both with the asset life-cycle and the manufacturing value chain) formalize this interconnectivity and realizes a uniform cloud service layer (Service Orchestration Cloud Interface in Figure 7) where heterogeneous factory assets and computing functionalities can coexist and be provided in a homogeneously virtualized manner.

\subsection{Case study preliminary design}

In this section, the preliminary architecture of a prototype smart factory is presented as a case study, demonstrating the applicability of the theoretical results. One of the main goals of the project is the realization of an intelligent Manufacturing Execution and Support System (MESS). The project is in the phase of defining, assembling and establishing the integration guidelines for a next generation, heterogeneous, collaborative I4.0 CPPSs (Figure 8). Further details will be published in a separate paper later on.

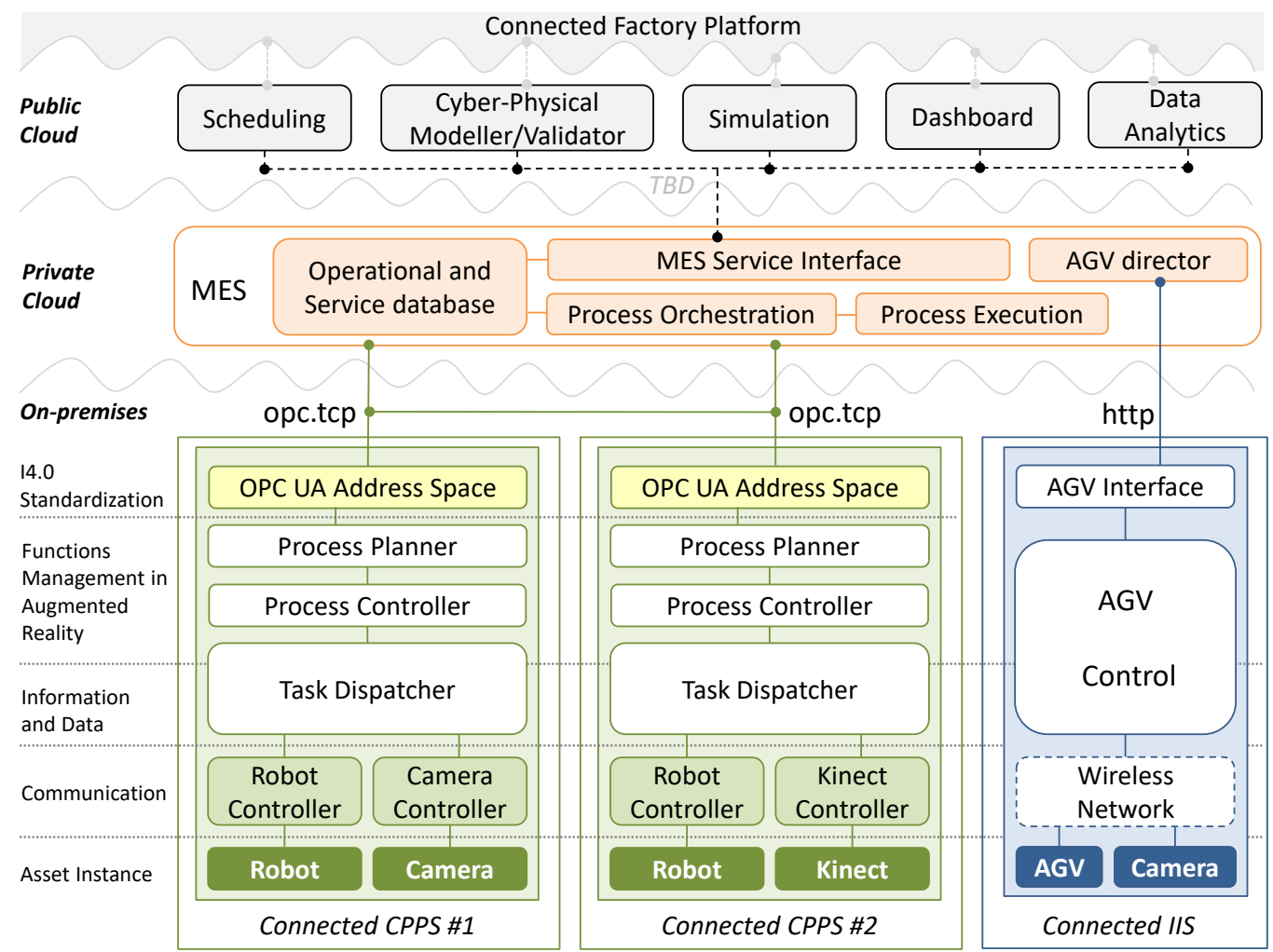

Figure 8: Case study preliminary architecture of RAMI 4.0 and IIRA virtualized and connected services/assets in GINOP Project's Manufacturing Execution and Support System 
The case study focuses on the implementation and standardization of digitalized manufacturing assets and services in a connected prototypical factory. The basic paradigm for machine-to-machine and the human-to-machine collaboration in the project is centered around the concept of the CPPS, an entity that, strictly coupled with its Digital Twin representation, enables the creation of adaptive production systems. The main factory deployment layers are depicted in Figure 8 and major architectural components are reported hereafter.

Public Cloud: it encompasses architectural components dedicated to the development and integration of enterprise-level manufacturing services (process simulation, DT of the CPPSs and 3D visualization and validation of the manufacturing environment, data analysis, scheduling of production activities, and so forth).

Private Cloud: it represents the actual deployment layer of the MESS, whose main functionality comprises the management and persistence of operational and service data, the orchestration (adapting scheduled activities to real-time events emerging from production) and execution (governing and directing) of manufacturing processes, as well as the interface to both upper and lower layer of the architecture.

On-premises production system: it focuses on the realization of standardized and connected of I4.0 components (CPPSs whose physical assets may be robots, web cameras, Kinect controllers, and an IIS intended for automated guided vehicles domain - AGVs - and orientation cameras). Each CPPS is the realization of the RAMI 4.0 AS and embodies the instantiation of its physical resources and provides capabilities related to communication, information and data, management of operations in augmented reality (CPPS layers on Figure 8), adopting a unified OPC UA modelling approach on top of the I4.0 components stack. A CPPS in the case study consists of autonomous and cooperative elements and sub-systems, connected with each other in a context-aware manner:

- the physical resources (asset instances);

- the cyber component (robot, camera and controllers), which is the interface on the "nearest" hardware with computing capacity and network connection; 
- the task dispatching controller is an asynchronous event based communication hub, where every CPPS is reachable in a standardized way and able to report its own status;

- the process planner is the adaptive virtualization of the environment to perform a functionality requested by the MESS;

- the process controller implements a precedence graph of tasks and executes them based on the CPPS status reports. A CPS is bound to its process components in terms of assigned resource, assigned functionality and assigned process precedence;

- Each CPPS functionality/service is modelled and provided via the Address Space of a dedicated OPC UA Server (interface details on Figure 8).

\section{Conclusions}

Cloud computing and cloud manufacturing became well known paradigms and many different applications have been developed over them. Despite this, the main challenges in cloud technology (like interoperability and security) still remain, especially due to the evolution and application of cloud-based (I)IoT systems which comprise of an enormous volume of heterogeneous connected devices, tools and machines. There is a strong demand for new system architectures, in order to address new emerging industrial requirements for globally interconnected structures. In this paper two significant frameworks, IIRA and RAMI 4.0, have been compared with each other and the latter with respect to OPC UA. The authors have derived common functional and modelling similarities in the interoperability and virtualization layers of each architecture and provided an example of how manufacturing services can be conceptualized and orchestrated in both architectures and provided to the external world via a common cloud-based interface. A preliminary architecture for a project case study has also been presented, in which the practical applicability of theoretical results has been introduced. An interpretation has been provided, too, of how a combination of IIC and RAMI 4.0 architectural guidelines may find relevant coexistence in end-to-end and complementary IIoT solutions. This observation can generate further discussions on a broader context of integrated industrial IoT solutions. Interoperability remains a central problem in the industrial implementations of new ICT paradigms and significant efforts have to be invested both in the fields of 
research and industrial applications, as this approach will probably be the key to realize the advantages promised by Industry 4.0 scenarios.

\section{Acknowledgements}

This research has been supported partially by the European Commission through the EU H2020 projects EXCELL under grant No. 691829 and GINOP-2.3.2-15-2016-00002.

\section{References}

Bommadevara, N., Kaplan, J., Starikova, I., 2016. Leaders and laggards in enterprise cloud infrastructure adoption. http://www.mckinsey.com/ business-functions / digital-mckinsey / our-insights / leaders- and- laggardsin-enterprise-cloud-infrastructure-adoption, [Retrieved April 23, 2018].

Chen, D., Doumeingts, G., Vernadat, F., 2008. Architectures for enterprise integration and interoperability: Past, present and future. Comput. Ind. 59 (7), 647-659.

Cloud-standards.org, 2018. The cloud standards wiki. http://cloudstandards.org, [Retrieved April 23, 2018].

EC, 2017. European commission sets out path to digitise european industry. https://ec.europa.eu/digital-single-market/en/news/commission-setsout-path-digitise-european-industry, [Retrieved April 23, 2018].

Fabode, S., 2016. New Business Models For IoT and IIoT Businesses. https: / / medium.com/startup-grind/new- business- models-for-iot- andiiot-businesses-2e5177d11a4a, [Retrieved April 23, 2018].

Gøtze, J., 2016. Reference architectures for Industry 4.0. https:// coe. qualiware.com/reference-architectures-for-industry-4-0/, [Retrieved April $23,2018]$.

Hein, A., 2017. Prepare for the new era of IIoT using OPC UA connectivity. https://www.honeywellprocess.com/library/news-and-events / presentations/hon-emea17-prepare-for-the-new-era-of-iiot-using-opc-uaconnectivity.pdf, [Retrieved April 23, 2018]. 
IIC \& Plattform Industrie 4.0, 2017. Industrial Internet Consortium \& Platform Industrie 4.0. https://www.infosys.com/engineering-services/whitepapers/Documents/industrial-internet-consortium-architecture.pdf, [Retrieved April 23, 2018].

Industrial Internet Consortium, 2015. Industrial Internet Reference Architecture. https://www.iiconsortium.org/IIRA.htm, [Retrieved April 23, 2018].

Infosys \& IIC, 2015a. IIC - Asset Efficiency Testbed. http://www . iiconsortium.org/asset-efficiency.htm, [Retrieved April 23, 2018].

Infosys \& IIC, 2015b. IIC - Industrial Digital Thread Testbed. http://www. iiconsortium.org/asset-efficiency.htm, [Retrieved April 23, 2018].

Lee, E. A., 2008. Cyber physical systems: Design challenges. Tech. Rep. UCB/EECS-2008-8, EECS Department, University of California, Berkeley.

Li, B.-H., Zhang, L., Wang, S.-L., Tao, F., Cao, J., Jiang, X., Song, X., Chai, X., 2010. Cloud manufacturing: a new service-oriented networked manufacturing model. Computer integrated manufacturing systems 16 (1), $1-7$.

Liu, Y., Xu, X., Oct 2016. Industry 4.0 and cloud manufacturing: A comparative analysis. Journal of Manufacturing Science and Engineering 139 (3), 034701-034701-8.

Marston, S., Li, Z., Bandyopadhyay, S., Zhang, J., Ghalsasi, A., 2011. Cloud computing the business perspective. Decision Support Systems 51 (1), 176 $-189$.

Mourad, M., Nassehi, A., Schaefer, D., 2016. Interoperability as a key enabler for manufacturing in the cloud. Procedia CIRP 52 (Supplement C), 30 - 34, the Sixth International Conference on Changeable, Agile, Reconfigurable and Virtual Production (CARV2016).

Ning, L., Xiaoping, L., 2012. A resource virtualization mechanism for cloud manufacturing systems. In: van Sinderen, M., Johnson, P., Xu, X., Doumeingts, G. (Eds.), Enterprise Interoperability. Springer Berlin Heidelberg, Berlin, Heidelberg, pp. 46-59. 
NSF, 2016. Cyber-Physical Systems. https://www.nsf.gov/pubs/2017/ nsf17529/nsf17529.pdf, [Retrieved April 23, 2018].

OPC Foundation, 2015. OPC Unified Architecture. https://opcfoundation. org/developer-tools/specifications-unified-architecture, [Retrieved April 23, 2018].

Pai, M., 2017. Interoperability between IIC Architecture and Industry 4.0 Reference Architecture for Industrial Assets. https://www.infosys. com/engineering-services / white- papers / Documents / industrial-internetconsortium-architecture.pdf, [Retrieved April 23, 2018].

Panetto, H., Molina, A., 2008. Enterprise integration and interoperability in manufacturing systems: Trends and issues. Comput. Ind. 59 (7), 641-646.

Schwab, K., 2015. The fourth industrial revolution: What it means and how to respond. https://www.foreignaffairs.com/articles/2015-12-12/fourthindustrial-revolution, [Retrieved April 23, 2018].

Uslnder, T., Epple, U., 2015. Reference model of industrie 4.0 service architectures. at - Automatisierungstechnik 63, 858-866.

VDI, VDE, ZVEI, 2015. Gma status report: Reference architecture model industrie 4.0 (rami 4.0). https://www.zvei.org/fileadmin/user_upload/ Presse_und_Medien / Publikationen / 2016 / januar / GMA_Status_Report_Reference_Archtitecture_Model_Industrie_4.0__RAMI_4.0_/GMA-StatusReport-RAMI-40-July-2015.pdf, [Retrieved April 23, 2018].

Wang, V. X., Xu, X. W., 2013. An interoperable solution for cloud manufacturing. Robotics and Computer-Integrated Manufacturing 29 (4), 232-247. URL http://dx.doi.org/10.1016/j.rcim.2013.01.005

Weins, K., 2017. Cloud computing trends: 2017 state of the cloud survey. https://www .rightscale.com/blog/cloud-industry-insights / cloudcomputing-trends-2017-state-cloud-survey, [Retrieved April 23, 2018].

Weissberger, A., 2011. IEEE CIO Says Cloud Interoperability a Bigger Problem than Security. http://techblog.comsoc.org/2011/06/, [Retrieved April $23,2018]$. 
Wu, D., Rosen, D. W., Wang, L., Schaefer, D., 2014. Cloud-based manufacturing: Old wine in new bottles? Procedia CIRP 17 (Supplement C), 94 -99, variety Management in Manufacturing.

Xu, X., 2012. From cloud computing to cloud manufacturing. Robotics and Computer-Integrated Manufacturing 28 (1), 75-86.

Yadgarova, Y., Taratukhin, V., 2016. An Interoperable Cloud Environment of Manufacturing Control System. Springer International Publishing, Cham, pp. $3-12$.

Zhang, L., Luo, Y., Tao, F., Li, B., Ren, L., Zhang, X., Guo, H., Cheng, Y., Hu, A., Liu, Y., 2012. Cloud manufacturing: A new manufacturing paradigm. Enterprise Information Systems 8, 1-21.

Zhang, Y., Zhang, G., Liu, Y., Hu, D., Jun. 2017. Research on services encapsulation and virtualization access model of machine for cloud manufacturing. J. Intell. Manuf. 28 (5), 1109-1123.

Zissis, D., Lekkas, D., 2012. Addressing cloud computing security issues. Future Gener. Comput. Syst. 28 (3), 583-592. 